This item was submitted to Loughborough's Research Repository by the author.

Items in Figshare are protected by copyright, with all rights reserved, unless otherwise indicated.

\title{
Speed profiles in wheelchair court sports; comparison of two methods for measuring wheelchair mobility performance
}

\section{PLEASE CITE THE PUBLISHED VERSION}

http://dx.doi.org/10.1016/j.jbiomech.2017.10.040

\section{PUBLISHER}

(C) Elsevier

VERSION

AM (Accepted Manuscript)

\section{PUBLISHER STATEMENT}

This work is made available according to the conditions of the Creative Commons Attribution-NonCommercialNoDerivatives 4.0 International (CC BY-NC-ND 4.0) licence. Full details of this licence are available at: https://creativecommons.org/licenses/by-nc-nd/4.0/

\section{LICENCE}

CC BY-NC-ND 4.0

\section{REPOSITORY RECORD}

van der Slikke, Rienk, Barry S. Mason, Monique Berger, and Victoria L. Goosey-Tolfrey. 2019. "Speed Profiles in Wheelchair Court Sports; Comparison of Two Methods for Measuring Wheelchair Mobility Performance". figshare. https://hdl.handle.net/2134/27379. 
5

6

\author{
R.M.A van der Slikke ${ }^{a,{ }^{\star}}$, B.S. Mason ${ }^{b}$, M.A.M. Berger ${ }^{a}$ \& V.L. Goosey-Tolfrey ${ }^{b}$
}

7

8 a Human Kinetic Technology, The Hague University of Applied Sciences,

9 Johanna Westerdijkplein 75, 2521EN The Hague,The Netherlands

$10 \mathrm{~b}$ The School of Sport Exercise and Health Sciences, Peter Harrison Centre for Disability Sport,

11 Loughborough University, UK

12

13

14

15

16

17

18 * Corresponding author at:

19 Human Kinetic Technology,

20 The Hague University of Applied Sciences,

21 Johanna Westerdijkplein 75, 2521EN, The Hague, The Netherlands.

22 Tel. +31704458704 .

23 E-mail address: r.m.a.vanderslikke@hhs.nl (R.M.A van der Slikke) 


\title{
for measuring wheelchair mobility performance
}

\author{
Rienk van der Slikke ${ }^{1}$, Barry Mason ${ }^{2}$, Monique Berger ${ }^{1}$ and \\ Vicky Goosey-Tolfrey² \\ ${ }^{1}$ The Hague University of Applied Sciences, The Netherlands \\ ${ }^{2}$ The School of Sport Exercise and Health Sciences, Peter Harrison Centre for Disability \\ Sport, Loughborough University, UK
}

\section{Abstract}

Wheelchair mobility performance is an important aspect in most wheelchair court sports, commonly measured with an indoor tracking system or wheelchair bound inertial sensors. Both methods provide key wheelchair mobility performance outcomes regarding speed. In this study, we compared speed profiles of both methods to gain insight into the level of agreement, for recommendations regarding future performance measurement.

Data were obtained from 5 male highly trained wheelchair basketball players during match play. Players were equipped simultaneously with a tag on the footplate for the indoor tracking system $(\sim 8 \mathrm{~Hz})$ and inertial sensors on both wheels and frame $(199.8 \mathrm{~Hz})$. Being part of a larger study on 3 vs 3 player game formats, data were collected in several matches with varying field sizes, but activity profiles closely resembled regular match play. Both systems provide similar outcomes regarding distance covered and average speed. Due to differences in sampling frequency and sensor location (reference point) on the wheelchair (for speed calculation), minor differences were revealed at low speeds $(<2.5 \mathrm{~m} / \mathrm{s})$. Since both systems provide complementary features, a hybrid solution as proved feasible in this study, could possibly serve as the new gold standard for mobility performance measurement in wheelchair basketball or wheelchair court sports in general.

Key words: wheelchair basketball, activity profiles, wheelchair mobility performance, inertial sensors, indoor tracking. 


\section{Introduction}

Quantitative assessment of an athlete's individual wheelchair mobility performance is needed to evaluate game performance, improve wheelchair settings and optimize training routines (Mason et al., 2013). Next to sport specific mobility performance outcomes, speed is one of the key performance indicators, relevant to all wheelchair sports (Burton et al., 2010, Rhodes et al., 2015 \& van der Slikke et al., 2016a). Based on a semi-structured interview of nine elite athletes, Mason et al. (2010) identified speed as one of the key performance indicators, important for optimizing wheelchair configuration. Fuss et al. (2012) emphasises the benefits of standard speed measurements in high-performance sports with decreasing costs of technology required. On court wheelchair mobility performance research, is often based on methods that either rely on wheelchair mounted or global reference sensors. Wheelchair bound systems essentially measure wheel rotational speed to calculate forward speed, with data loggers based on reed-switches (Tolerico et al., 2007), potentiometers (Velocometer, Moss et al., 2003) or inertial sensors (Pansiot et al., 2011 \& van der Slikke et al., 2015a). If sensors are placed in a fixed global position, wheelchair speed is measured with either laser technology (Ferro et al., 2016) or radio frequency based technology (Rhodes et al., 2014). This technical note describes the comparison between two common systems for performance measurement in court sports, namely the inertial sensor based wheelchair mobility performance monitor (WMPM, van der Slikke et al., 2015a) and the global reference based indoor tracking system (ITS, Rhodes et al., 2014). Inertial sensor based methods like the WMPM allow for easy and accurate measurement of wheelchair mobility performance, but provide no information about absolute field position. Indoor tracking systems provide positional data, enabling tactical team analyses, but lack the option to calculate higher order outcomes like acceleration, due to sample frequency restrictions. In this study, we compared outcomes of both methods regarding speed, to gain insight into the level of agreement between devices. 


\section{Methods}

\section{Participants \& instrumentation}

Five male, highly trained wheelchair basketball players (age: $20 \pm 1$ years; playing experience: $7 \pm 2$ years, IWBF classification: $1.0,2.0,3.0,3.5 \& 4.5)$ volunteered to participate in the study. Their wheelchair mobility performance was monitored using an ITS (Ubisense, $\sim 8 \mathrm{~Hz}$ ) with a tag positioned on the footplate and simultaneously with three inertial sensors (Shimmer3, $199.8 \mathrm{~Hz}$ ) on wheels and frame (WMPM) of their own customised sports wheelchairs. Since the objective was to compare existing technologies, procedures and settings used for ITS and WMPM were in line with previous research. Measurements and setup

Being part of a larger study on wheelchair basketball game innovations (Mason et al., 2017), measurements (6 times 10 min.) were performed during different 3 versus 3 game formats (full court, half court and a modified court length of $22 \mathrm{~m}$ ). Six ITS sensors were located around the perimeter of a regulation-size wheelchair basketball court $(28 \times 15 \mathrm{~m})$. The sensors were positioned at each of the four corners of the court, with two additional sensors positioned at the half-way line. Each sensor was mounted on an extendable tripod, elevated approximately $4 \mathrm{~m}$ high. The digital signal processing of the ITS was originally optimised for position accuracy, using a 3-pass sliding-average filter with a window width proportional to the tag frequency (Rhodes et al., 2014). In the ITS processing for this study, a five point $(\sim 0.625 \mathrm{~Hz})$ sliding average filter was applied to the raw position data of the tag. The tag was positioned at the footplate to ensure best reception by the sensors, as described by Perrat et al. (2015). For the wheelchair mobility profile, speed is derived from the filtered position data. Note that the outcomes of the ITS describe the motion of the tag mounted on the footplate, whereas the WMPM describes the movement of the wheelchair frame centre in-between both main wheels, so the reference points on the wheelchair differ (Figure 1). For the WMPM speed calculation is based on wheel rotation derived from the wheel sensors, with additional skid correction algorithm (van der Slikke 2015b). Heading direction is based on the inertial sensor mounted to the frame (van der Slikke 2015a). Due to the shared frequency bandwidth 
between multiple player tags in the ITS, the sample frequency varied slightly around $8 \mathrm{~Hz}$.

109 Sample timestamps were utilized to resample up to the WMPM frequency (linear

110 interpolation, Interp1, Matlab). Given the absence of hardware synchronisation options, a

111 cross-correlation of speed signals was used for post synchronisation of systems (Li et al., 112 1999).

\section{Data processing}

114 For each of the six measurements per player (10 min. match play), distance covered, speed 115 and time in six fixed speed zones (see Table 1) was calculated. The speed zone thresholds 116 are enclosed in the ITS method, originally based on the research regarding wheelchair rugby 117 (Rhodes et al., 2015) and wheelchair tennis (Sindall et al., 2013).

118 The single tag per wheelchair for the ITS does not allow for determination of heading 119 direction of the wheelchair, so no distinction between forward and backward movement is 120 made. The WMPM does differentiate between directions, but to allow for proper comparison 121 with the ITS, absolute values of speed were used. To gain insight in the relationship between 122 ITS and WMPM across speeds, the average value of both systems categorised by $0.05 \mathrm{~m} / \mathrm{s}$ 123 increments, were plotted against each other.

124 Although the WMPM reference point at the frame centre seems preferable over a reference 125 point at the foot plate, the ITS position outcome does not allow for recalculation of an 126 alternative point on the wheelchair frame, since heading direction is unknown. It was 127 however possible to re-calculate WMPM outcomes to a foot plate reference point and with 128 filtering similar to the ITS procedure. The WMPM heading direction and the measured 129 distance between rear axle and foot plate was used to calculate the speed of the footplate 130 reference point (see Appendix I). This speed signal was low-pass filtered $\left(0.5 \mathrm{~Hz}, 2^{\text {nd }}\right.$ order 131 butterworth) and used to calculate the alternative outcomes, named WMPM2. This is not the 132 preferred outcome of the WMPM, but does allow for the most optimal comparison of 133 calculated displacement and speed. 


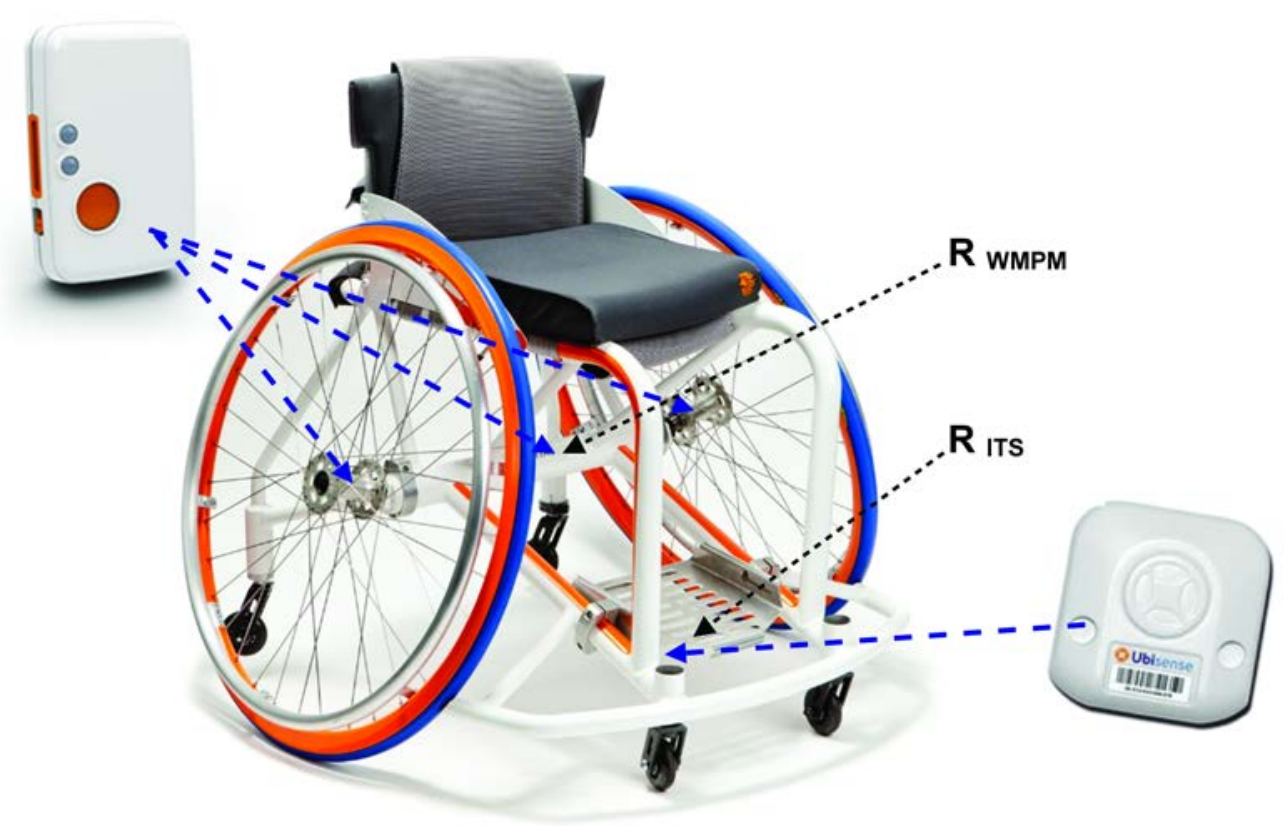

134

$\underline{\text { Table } 1}$

137 Average speed and distance related outcomes of the five athletes in six measurements. Data of the

indoor tracking system (ITS) are shown in the middle, the Wheelchair Mobility Performance Monitor

139 (WMPM) outcomes are shown on the left and the adjusted WMPM2 shown on the right. Columns in-

140 between show the average differences and standard deviations (SD) of the differences between

141 methods. The lower part shows the percentage time spend in the different speed zones, as adopted

from Mason et al. (2014).

143

\begin{tabular}{|c|c|c|c|c|c|c|c|c|}
\hline & & WMPM & difference & $\underline{S D}$ & $\underline{\underline{\mathrm{TTS}}}$ & difference & $\underline{\underline{S D}}$ & WMPM2 \\
\hline Distance & & 837.8 & $-2.6 \%$ & $3.2 \%$ & 882.3 & $0.1 \%$ & $3.3 \%$ & 883.4 \\
\hline \multirow{2}{*}{ Speed $(\mathrm{m} / \mathrm{s})$} & average & $\underline{1.30}$ & $-2.6 \%$ & $3.2 \%$ & 1.37 & $0.1 \%$ & $3.3 \%$ & 1.38 \\
\hline & $\underline{\text { RMSE }}$ & & $\underline{0.41}$ & $\underline{0.060}$ & & $\underline{0.33}$ & $\underline{0.072}$ & \\
\hline \multirow{6}{*}{$(\mathrm{m} / \mathrm{s})$} & $0-0.5$ & $22.4 \%$ & 13.7 & 5.1 & $8.7 \%$ & 5.7 & $\underline{4.5}$ & $14.4 \%$ \\
\hline & $\underline{0.5-1.5}$ & $\underline{37.9 \%}$ & $\underline{-15.7}$ & $\underline{5.9}$ & $53.6 \%$ & $\underline{-9.0}$ & $\underline{5.1}$ & $44.6 \%$ \\
\hline & $\underline{1.5-2.5}$ & $\underline{29.3 \%}$ & $\underline{-0.1}$ & 3.2 & $\underline{29.4 \%}$ & $\underline{2.0}$ & $\underline{2.8}$ & $31.3 \%$ \\
\hline & $2.5-3.0$ & $\underline{6.6 \%}$ & $\underline{1.0}$ & $\underline{1.4}$ & $5.5 \%$ & $\underline{0.9}$ & $\underline{1.4}$ & $\underline{6.4 \%}$ \\
\hline & $\underline{3.0-3.5}$ & $\underline{2.8 \%}$ & $\underline{0.7}$ & $\underline{0.9}$ & $\underline{2.1 \%}$ & $\underline{0.4}$ & $\underline{0.9}$ & $\underline{2.5 \%}$ \\
\hline & $\underline{3.5+}$ & $\underline{\underline{1.0 \%}}$ & $\underline{\underline{0.3}}$ & $\underline{0.7}$ & $\underline{0.7 \%}$ & $\underline{0.0}$ & $\underline{0.7}$ & $\underline{0.7 \%}$ \\
\hline
\end{tabular}




\section{Results}

148 The average distance calculated per $10 \mathrm{~min}$. game time was $882.3 \mathrm{~m}$ for the ITS, $837.8 \mathrm{~m}$ for 149 the WMPM and 883.4 $\mathrm{m}$ for WMPM2 (see Table 1). Differences in calculated distance per 10 150 min. match play, between ITS and WMPM ranged from $-7.6 \%$ to $6.4 \%$ and between ITS and 151 WMPM2 from $-7.6 \%$ to $7.3 \%$. The root mean square differences (RMSDs) were calculated 152 based on the comparison between the resampled ITS speed signal versus the WMPM speed 153 (RMSD of $0.41 \mathrm{~m} / \mathrm{s}$ ) and the WMPM2 speed (RMSD of $0.33 \mathrm{~m} / \mathrm{s}$ ). The differences in 154 percentage time spent within the six fixed speed zones varied from $0.1-15.7$ between ITS 155 and WMPM and 0.0 - 9.0 between ITS and WMPM2 (see Table 1). Figure 2 shows a typical example (20s game play) of the speed of a wheelchair as measured with the different systems. The average ITS corresponding speed per $0.05 \mathrm{~m} / \mathrm{s}$ speed category of the WMPM 158 is shown in Figure 3.

$\underline{\text { Table } 1}$

Average speed and distance related outcomes of the five athletes in six measurements. Data of the indoor tracking system (ITS) are shown in the middle, the Wheelchair Mobility Performance Monitor (WMPM) outcomes are shown on the left and the adjusted WMPM2 shown on the right. Columns inbetween show the average differences and standard deviations (SD) of the differences between methods. The lower part shows the percentage time spend in the different speed zones, as adopted from Mason et al. (2014).

\begin{tabular}{|c|c|c|c|c|c|c|c|c|}
\hline & & WMPM & difference & $\underline{S D}$ & $\underline{\underline{\text { ITS }}}$ & difference & $\underline{S D}$ & WMPM2 \\
\hline Distance & & $\underline{837.8}$ & $-2.6 \%$ & $3.2 \%$ & 882.3 & $0.1 \%$ & $3.3 \%$ & 883.4 \\
\hline \multirow{2}{*}{ Speed $(\mathrm{m} / \mathrm{s})$} & average & $\underline{1.30}$ & $-2.6 \%$ & $\underline{3.2 \%}$ & 1.37 & $\underline{0.1 \%}$ & $3.3 \%$ & $\underline{1.38}$ \\
\hline & RMSE & & $\underline{0.41}$ & $\underline{0.060}$ & & $\underline{0.33}$ & $\underline{0.072}$ & \\
\hline \multirow{6}{*}{$(\mathrm{m} / \mathrm{s})$} & $0-0.5$ & $22.4 \%$ & 13.7 & $\underline{5.1}$ & $8.7 \%$ & 5.7 & $\underline{4.5}$ & $14.4 \%$ \\
\hline & $0.5-1.5$ & $37.9 \%$ & -15.7 & $\underline{5.9}$ & $53.6 \%$ & -9.0 & $\underline{5.1}$ & $44.6 \%$ \\
\hline & $\underline{1.5-2.5}$ & $\underline{29.3 \%}$ & $\underline{-0.1}$ & 3.2 & $\underline{29.4 \%}$ & $\underline{2.0}$ & $\underline{2.8}$ & $31.3 \%$ \\
\hline & $2.5-3.0$ & $6.6 \%$ & 1.0 & 1.4 & $\underline{5.5 \%}$ & $\underline{0.9}$ & $\overline{1.4}$ & $\underline{6.4 \%}$ \\
\hline & $3.0-3.5$ & $2.8 \%$ & 0.7 & 0.9 & $2.1 \%$ & 0.4 & $\underline{0.9}$ & $2.5 \%$ \\
\hline & $3.5+$ & $1.0 \%$ & $\underline{\underline{0.3}}$ & $\underline{0.7}$ & $\underline{0.7 \%}$ & $\underline{0.0}$ & $\underline{0.7}$ & $\underline{0.7 \%}$ \\
\hline
\end{tabular}


$173 \quad$ Figure2
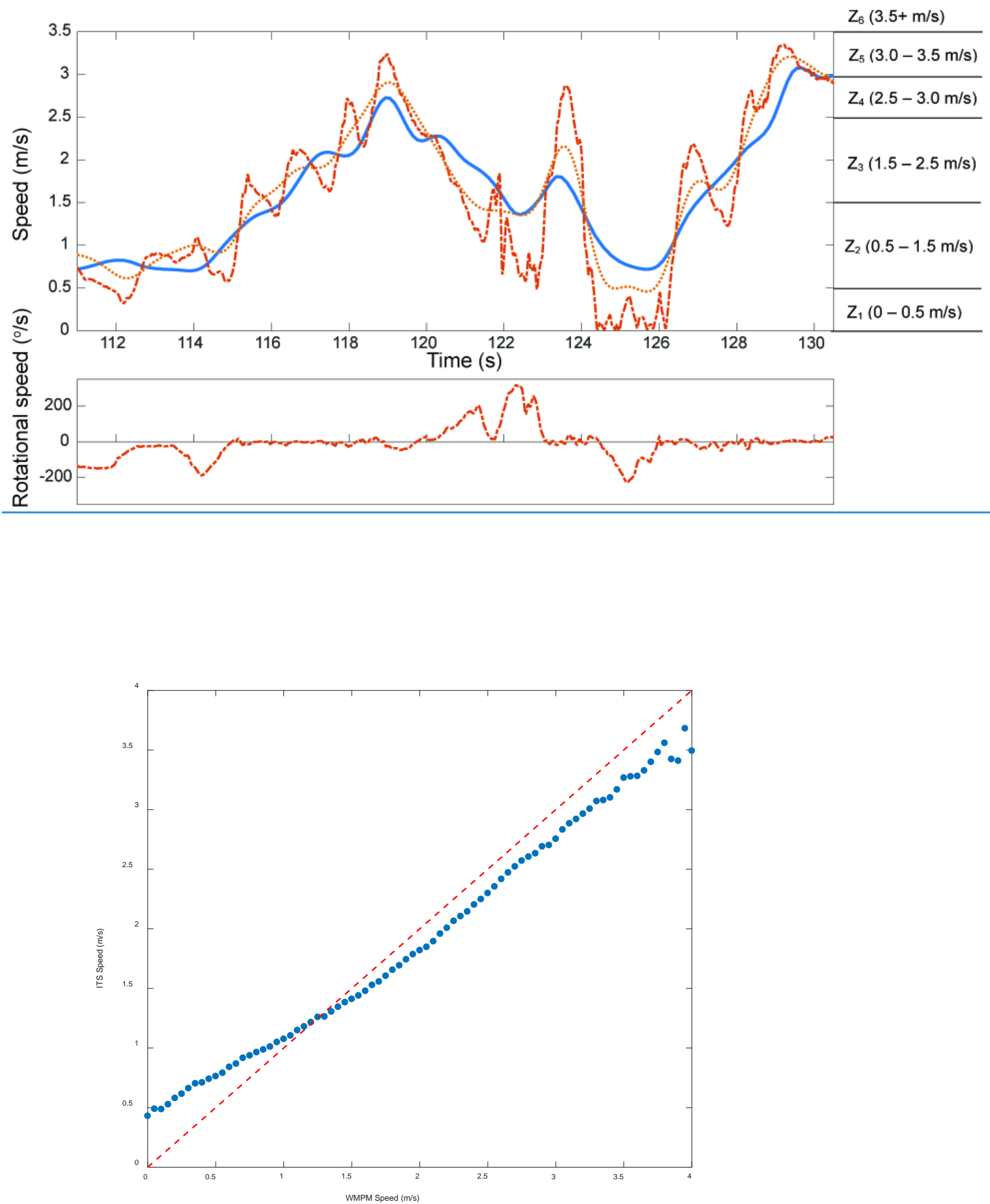

Figure 3 The ITS speed plotted against the WMPM speed. Per increment of $0.05 \mathrm{~m} / \mathrm{s}$ the average of all corresponding ITS 
Discussion

184

185

186

187

188

189

190

191

192

193

194

195

196

197

198

199

200

201

202

203

204

205

206

207

208

In general, both systems provide quite similar speed data, but the method features do account for some typical deviations. The difference in reference point on the wheelchair (footplate vs. frame centre) affected the calculated speed and distance slightly $(\leq 2.6 \%)$. In the ITS, turns on the spot (turning without displacement of the frame centre) will cause a displacement equal to the circumference path described by the footplate, whereas the WMPM will not calculate any displacement at the same time. Since the ITS only provides information on tag position and not on heading direction, it is impossible to calculate the speed and distance covered of a different reference point on the wheelchair. To attain a fair comparison, it is however possible to adjust the WMPM outcomes to a reference point near the footplate. Once adjusted, systems provide very similar distance and average speed data $(\leq 0.1 \% \pm 3.3 \%)$, although still individual differences up to $7.6 \%$ occur. The RMSD of 0.41 $\mathrm{m} / \mathrm{s}$ for the WSPM speed and $0.33 \mathrm{~m} / \mathrm{s}$ for the WMPM2 speed seem acceptable for this type of measurements, where speeds range from $0-\sim 5 \mathrm{~m} / \mathrm{s}$ in match play (van der Slikke et al., 2016). Differences in instantaneous speeds as expressed in the RMSD, do not influence the average speeds calculated, but might affect calculated maximal speeds. The position of the reference point causes a very low percentage of time in the lowest speed zone $(<0.5 \mathrm{~m} / \mathrm{s})$ for the ITS and WMPM2, because when not moving forward, often turns on the spot still cause some speed (see Figure 2, time 124.5 - 126s). The restricted sample frequency of the ITS, requires low-pass filtering with a very low cut-off frequency $(\sim 0.625 \mathrm{~Hz})$, drawing the speed signal towards the average, so with more time assigned to the corresponding average speed class $(0.5-1.5 \mathrm{~m} / \mathrm{s}$, see Figure 2). The abovementioned effects also show in Figure 3, with ITS values higher than WMPM in speeds below $\sim 1.5 \mathrm{~m} / \mathrm{s}$, due to the tag position and rotations, and ITS values slightly lower than the WMPM in speeds over $\sim 1.5 \mathrm{~m} / \mathrm{s}$, due to more severe low-pass filtering. These results provided an insight to what extent research outcomes obtained with both methods are interchangeable. For distance, average speed and 
above average speeds zones ( $>1.5 \mathrm{~m} / \mathrm{s}$ ), both methods provide similar outcomes. Speed profiles show higher ITS values for below average speeds and slightly lower values for above average speeds, compared to the WMPM.

Although match play settings for the measurements deviated slightly from regular 5 vs 5 match play at regular court settings, the activity profiles did closely resemble the typical elite level performance. The average speed in the measurements was $1.37 \mathrm{~m} / \mathrm{s}$ (1.3 for the WMPM), which is only slightly lower than reported in literature for elite level wheelchair basketball match play $1.48 \mathrm{~m} / \mathrm{s}$ (Sporner et al., 2009) and $1.57 \mathrm{~m} / \mathrm{s}$ (van der Slikke et al., 2016b). Also, peak speeds were a bit lower than reported earlier in elite wheelchair basketball, $2.19 \mathrm{~m} / \mathrm{s}$ compared to $2.95 \mathrm{~m} / \mathrm{s}$ (van der Slikke et al., 2016b). The somewhat lower average and peak speed could be explained by the reduced court sizes (half court and modified $22 \mathrm{~m}$ court length) in part of the measurements. Those dimensions might also have

221 led to an increase in rotations, magnifying the differences between systems due the difference in reference point. Regular match play with higher average speed and less rotations, is expected to positively influence method agreement.

\section{Conclusion}

For applied sports research, ease of use and fast turnaround of feedback are crucial in any method. Both measurement systems meet those demands and outcomes proved interchangeable to a great extent. The type of method used for future research is depending on the research question, with a focus on field position (ITS) or acceleration profiles (WMPM). The ITS provides information on field position, so enables wheelchair mobility performance analysis split by game specific characters (e.g. offence-defence, location to the bucket and heat maps). The WMPM provides more detailed kinematic data, allowing for analyses regarding e.g. accelerations, rotations and push characteristics (van der Slikke et al., 2016b). For the most comprehensive approach, this study proved the feasibility of a hybrid solution incorporating both methods, hence providing the best of both worlds and possibly serving as the new standard for mobility performance in court sports. 
238 Conflict of interest

239 None.

\section{References}

242 Burton, M., Fuss, F. K., \& Subic, A. (2010). Sports wheelchair technologies. Sports Technology, 3(3), $243 \quad 154-167$.

244 Ferro, A., Villacieros, J., \& Pérez-Tejero, J. (2016). Sprint performance of elite wheelchair basketball players: applicability of a laser system for describing the velocity curve. Adapted Physical Activity 246 Quarterly, 33(4), 358-373.

247 Fuss, F. K. (2012). Speed measurements in wheelchair sports-theory and application. Sports 248 Technology, 5(1-2), 29-42.

249 Li, L., \& Caldwell, G. E. (1999). Coefficient of cross correlation and the time domain 250 correspondence. Journal of Electromyography and Kinesiology, 9(6), 385-389.

251 Mason, B. S., Porcellato, L., van der Woude, L. H., \& Goosey-Tolfrey, V. L. (2010). A qualitative 252 examination of wheelchair configuration for optimal mobility performance in wheelchair sports: a pilot 253 study. Journal of Rehabilitation Medicine, 42(2), 141-149.

254 Mason, B. S., van der Woude, Lucas HV, \& Goosey-Tolfrey, V. L. (2013). The ergonomics of 255 wheelchair configuration for optimal performance in the wheelchair court sports. Sports Medicine, 256 43(1), 23-38.

257 Mason, B., Lenton, J., Rhodes, J., Cooper, R., \& Goosey-Tolfrey, V. (2014). Comparing the activity 258 profiles of wheelchair rugby using a miniaturised data logger and radio-frequency tracking 259 system. BioMed Research International, 2014.

260 Mason, B.S., van der Slikke, R.M.A., Berger, M.A.M. \& Goosey-Tolfrey, V. L. (2017). The effect of 261 small-sided game formats on physical and technical performance in wheelchair basketball. Journal of 262 Sports Physiology and Performance, conditionally accepted.

263 Moss, A. D., Fowler, N. E., \& Tolfrey, V. L. (2003). A telemetry-based velocometer to measure 264 wheelchair velocity. Journal of Biomechanics, 36(2), 253-257. 
Pansiot, J., Zhang, Z., Lo, B., \& Yang, G. Z. (2011). WISDOM: wheelchair inertial sensors for displacement and orientation monitoring. Measurement Science and Technology, 22(10), 105801. Rhodes, J., Mason, B., Perrat, B., Smith, M., \& Goosey-Tolfrey, V. (2014). The validity and reliability of a novel indoor player tracking system for use within wheelchair court sports. Journal of Sports Sciences, 32(17), 1639-1647.

Perrat, B., Smith, M. J., Mason, B. S., Rhodes, J. M., \& Goosey-Tolfrey, V. L. (2015). Quality assessment of an Ultra-Wide Band positioning system for indoor wheelchair court sports. Proceedings of the Institution of Mechanical Engineers, Part P: Journal of Sports Engineering and

273 Technology, 229(2), 81-91.

274 Rhodes, J. M., Mason, B. S., Perrat, B., Smith, M. J., Malone, L. A., \& Goosey-Tolfrey, V. L. (2015). 275 Activity profiles of elite wheelchair rugby players during competition. International Journal of Sports 276 Physiology and Performance, 10(3), 318-324.

277 Tolerico, M. L., Ding, D., Cooper, R. A., \& Spaeth, D. M. (2007). Assessing mobility characteristics and 278 activity levels of manual wheelchair users. Journal of Rehabilitation Research and 279 Development, 44(4), 561.

280 Sindall, P., Lenton, J. P., Whytock, K., Tolfrey, K., Oyster, M. L., Cooper, R. A., \& Goosey-Tolfrey, V. 281 L. (2013). Criterion validity and accuracy of global positioning satellite and data logging devices for 282 wheelchair tennis court movement. The journal of spinal cord medicine, 36(4), 383-393.

283 Sporner, M. L., Grindle, G. G., Kelleher, A., Teodorski, E. E., Cooper, R., \& Cooper, R. A. (2009). 284 Quantification of activity during wheelchair basketball and rugby at the National Veterans Wheelchair 285 Games: A pilot study. Prosthetics and Orthotics International, 33(3), 210-217.

286 Van Der Slikke, R. M. A., Berger, M. A. M., Bregman, D. J. J., Lagerberg, A. H., \& Veeger, H. E. J. 287 (2015a). Opportunities for measuring wheelchair kinematics in match settings; reliability of a three 288 inertial sensor configuration. Journal of Biomechanics, 48(12), 3398-3405.

289 Van der Slikke, R. M. A., Berger, M. A. M., Bregman, D. J. J., \& Veeger, H. E. J. (2015b). Wheel skid 290 correction is a prerequisite to reliably measure wheelchair sports kinematics based on inertial sensors. 291 Procedia Engineering, 112, 207-212.

292 Van der Slikke, R., Berger, M., Bregman, D., \& Veeger, D. (2016a). Push characteristics in wheelchair 293 court sport sprinting. Procedia Engineering, 147, 730-734. 
Van der Slikke, R. M. A., Berger, M. A. M., Bregman, D. J. J., \& Veeger, H. E. J. (2016b). From big data to rich data: The key features of athlete wheelchair mobility performance. Journal of

\section{Acknowledgement}

We would like to thank for the Loughborough University's Enterprise Projects Group and the Peter Harrison Foundation for funding this research and the valuable assistance of Mike Hutchinson during the measurements.

302

303

\section{Appendix I}

304 The frame centre displacement in the WMPM is based on the average wheel speed derived 305 from wheel rotational speed and wheel circumference (van der Slikke et al., 2015a). This 306 calculation results in a reference point in the middle between both main wheels, thus the 307 middle of the camber bar. To recalculate the speed of a reference point on the footplate, the 308 speed of this point due to rotations with regard to the original reference point, is added. See 309 Equation 1, with the recalculated speed (Speed ${ }_{\text {WMPM2, }}[\mathrm{m} / \mathrm{s}]$ ) based on the original speed 310 (Speed ${ }_{\text {wмPM }},[\mathrm{m} / \mathrm{s}]$ ), the frame rotational speed (RotSpeed ${ }_{\text {WMPM }},[\mathrm{rad} / \mathrm{s}]$ and the distance

311 between rear axle and footplate $\left(d_{a-f},[m]\right)$. 